\title{
Pinéale et rétine
}

Les phototransducteurs de la pinéale (épiphyse), et ceux de la rétine (cônes et bâtonnets) présentent des convergences au niveau : de leur organisation, de plusieurs types de molécules et des mécanismes des activités rythmiques nycthémérales. La synthèse nocturne de la mélatonine correspond à l'une de ces manifestations cycliques, contrôlée par des oscillateurs qui sont eux-mêmes sensibles à l'alternance de la lumière et de l'obscurité (photopériode). Chez les mammifères, la mélatonine élaborée par les pinéalocytes, phototransducteurs très modifiés mais très actifs, joue un rôle important dans le contrôle de rythmes biologiques et comportementaux.

\section{Jean-Pierre Collin Jean-Pierre Faure Jacky Falcón Pierre Voisin Paul Brisson Massoud Mirshahi}

\section{ADRESSES}

J.-P. Collin, J. Falcón, P. Voisin, P. Brisson : laboratoire de biologie cellulaire et Cnrs UA 290, université de Poitiers, 40, avenue du recteur Pineau, 86022 Poitiers Cedex, France. J.-P. Faure, M. Mirshahi : laboratoire d'immunopathologie de l'œil, Inserm U. 86, Hôtel Dieu, 1, place du parvis Notre-Dame,

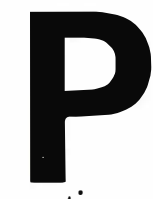
our vivre en harmonie avec l'environnement, l'homme et les autres vertébrés décodent divers types de signaux et informations d'origine externe. Les photorécepteurs de la rétine, les transducteurs de la pinéale et les " photorécepteurs encéphaliques profonds" sont sensibles aux signaux engendrés par les variations quotidiennes de l'éclairement. La notion de " photorécepteurs encéphaliques profonds", issue des célèbres travaux de Benoît sur la photo-gonadostimulation chez le canard, a été élargie à d'autres espèces [1]. En l'absence d'une identification précise de ces cellules, seuls les autres phototransducteurs diencéphaliques seront considérés ici.

Au cours du cycle lumière/obscurité de $24 \mathrm{~h}$ (ou photopériode), les phototransducteurs discriminent les variations d'intensité, de composition spectrale, de durée du stimulus lumineux et fournissent des réponses adéquates. Les signaux ainsi élaborés contribuent à l'optimisation des relations entre l'organisme et son environnement : d'une part, ils sont à l'origine d'une description ordonnée du monde extérieur (seuls les photorécepteurs rétiniens sont impliqués dans la vision) ; d'autre part, ils participent aux mécanismes de synchronisation, sur la photopériode (information temporelle), des rythmes journaliers et saisonniers. De telles rythmicités, sous contrôle d'horloges (ou oscillateurs) internes à l'organisme, retentissent à la fois sur le comportement et les fonctions physiologiques, et d'une façon plus générale sur les activités métaboliques.

L'étude des mécanismes de cette synchronisation fait actuellement apparaître des analogies et identités malgré la diversité des phototransducteurs. Les acquis portant sur les mécanismes de la phototransduction, et sur la sécrétion nocturne et le rôle de la mélatonine (expression de l'activité rythmique des phototransducteurs) débouchent sur d'importantes perspectives médicales.

\section{Organisation fonctionnelle}

Les photorécepteurs rétiniens (PR), bâtonnets et cônes, présentent quatre compartiments : (a) un segment externe - cil modifié qui différencie un empilement important de saccules aplatis (ou disques), limités par une membrane - siège de la phototrans- 


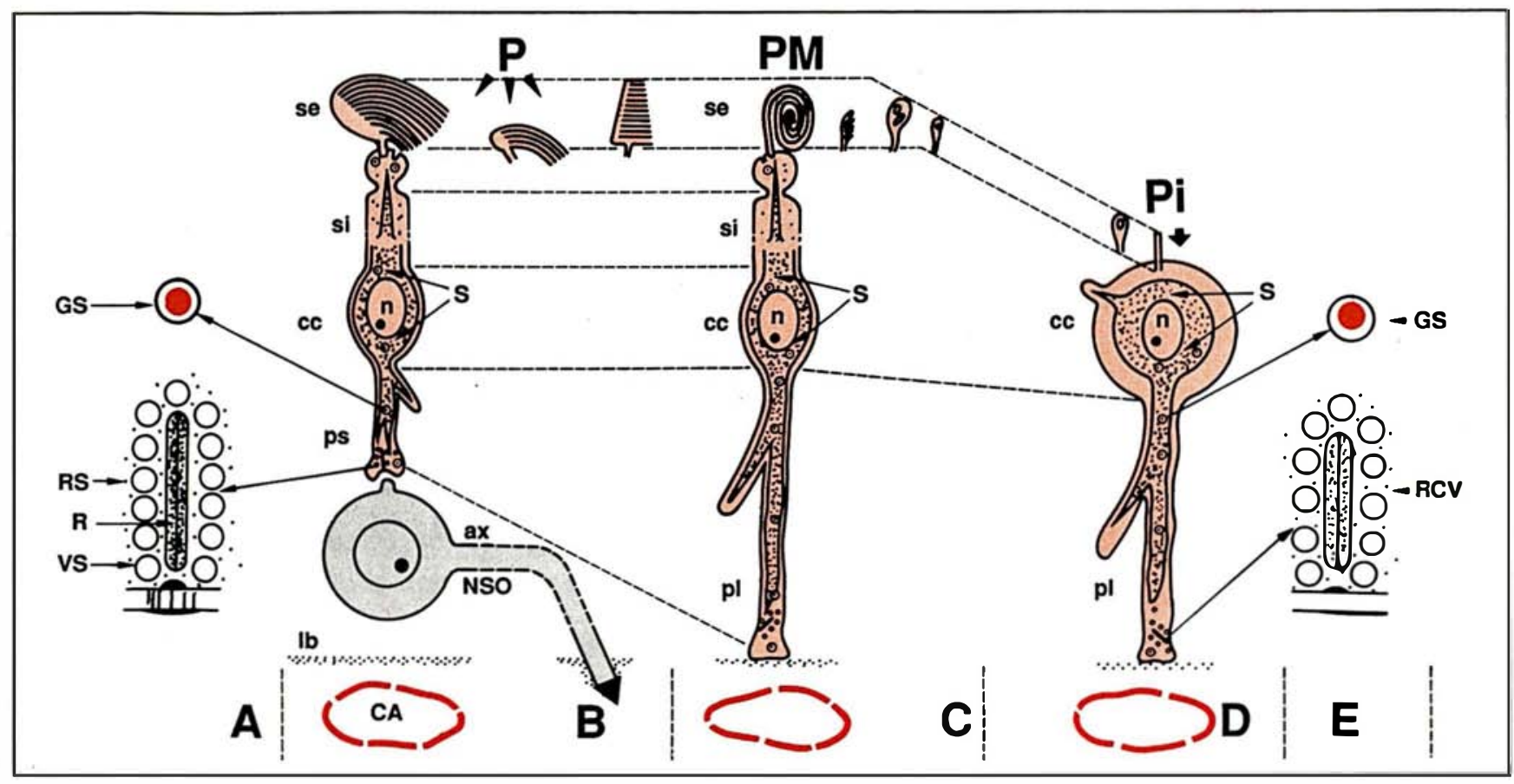

Figure 1. "Spectre " des transducteurs dans l'organe pinéal des vertébrés. A. Représentation schématique d'un grain de sécrétion (GS) et de rubans synaptiques (RS) dans les photorécepteurs (voir $B$ ). $R=$ ruban; VS = vésicule synaptique. B. Photorécepteur (P) connecté synaptiquement à un neurone de second ordre (NSO). ax = axone; $C A=$ capillaire $; c c=$ corps cellulaire $; l b=$ lame basale $; n=$ noyau $; p s=$ pédicule synaptique $; S=$ système endomembranaire (localisation du réticulum endoplasmique, des dictyosomes et des grains de sécrétion); se = segment externe; si = segment interne. C. Photorécepteur modifié (PM). pl = prolongement asynaptique. D. Pinéalocyte (Pi). E. Grain de sécrétion (GS) et ruban circonscrit de vésicules (RCV) à rôle inconnu dans les Pi ou les PM.

duction; (b) un segment interne sur lequel s'insère le segment externe, et contenant les organites produisant l'énergie et ceux responsables de l'élaboration des macromolécules et des membranes ; (c) un corps cellulaire (avec le noyau) connecté (d) au pôle neurotransmetteur, lui-même relié, par des synapses de type ruban, aux cellules bipolaires et horizontales [2, 3]. Les bâtonnets se distinguent des cônes principalement par la structure des disques, la nature du photopigment et l'organisation synaptique.

Dans l'organe pinéal des poïkilothermes, les photorécepteurs (P) - montrant des analogies structurales avec les cônes rétiniens sont connectés synaptiquement aux neurones de $2^{\mathrm{e}}$ ordre qui projettent leurs axones vers le cerveau (figure $1 A-B$ ) [4-6]. Les $\mathrm{P}$ subissent d'importants remaniements au cours de l'évolution. Ainsi, les photorécepteurs modifiés (PM) (figure 1C) des reptiles et des $m / s n^{\circ} 1$ ool. 4, janoier 88 oiseaux, à segment externe atypique ou rudimentaire, conservent dans bon nombre de cas la photosensibilité mais perdent leurs connexions synaptiques avec les neurones de $2^{e}$ ordre ; leurs prolongements asynaptiques se terminent à proximité de capillaires. Enfin, chez les mammiferes, dont l'homme, le pinéalocyte $(\mathrm{Pi})$ (figure $1 D)$ se réduit à un corps cellulaire et à des prolongements, en majorité orientés vers les capillaires. Les homologies de structure entre ces trois principaux types cellulaires ainsi que la présence de molécules communes ont conduit à proposer l'existence d'un "spectre " (ou lignée) de transducteurs pinéaliens [4-6]. Le Pi est définitivement considéré comme un dérivé de photorécepteur dont la photosensibilité et la neurotransmission ont disparu, au moins chez une majorité d'espèces.

Dans le spectre visible, les PR et les $\mathrm{P}$ different par leur sensibilité aux intensités de l'éclairement (luminances) et par les propriétés d'absorption de leurs photopigments (bâtonnets : valeurs faibles de la luminance, $\lambda$ max : environ $500 \mathrm{~nm}$; cônes : valeurs élevées de la luminance, $\lambda \max : 419,531$ et $559 \mathrm{~nm}$ chez l'homme; $P$ : valeurs faibles à élevées de la luminance, $\lambda$ max : 500 à $540 \mathrm{~nm}$ et parfois au-delà de $600 \mathrm{~nm}$ ) [2, $7,8]$. Les $P$ de la pinéale sont, en plus, sensibles à l'ultraviolet. Dans la rétine, les bâtonnets sont impliqués dans la vision crépusculaire (ou scotopique) et les cônes dans la vision photopique et la perception des couleurs. Les $P$, non impliqués dans la perception des couleurs, sont quant à eux des indicateurs de la durée du jour et des dosimètres des radiations solaires [7, 8].

\section{Phototransduction}

Protéines de la phototransduction. Le lecteur pourra se reporter à une revue parue ici [9] sur 
les mécanismes de la phototransduction - au niveau des segments externes des bâtonnets mettant en jeu : la rhodopsine, la transducine, la phosphodiestérase du GMPc (guanosine monophosphate cyclique), la rhodopsinekinase, l'antigène-S ( = arrestine). A l'obscurité, la liaison du GMPc ( $2^{e}$ messager) à des canaux sodiques maintient ces canaux ouverts et dépolarise la membrane. Au contraire, l'énergie photonique engendre une cascade de réactions (impliquant les protéines de la phototransduction), conduisant à l'hydrolyse du GMPc et à l'hyperpolarisation de la membrane, par fermeture des canaux sodiques.

Des mécanismes assez voisins pourraient se retrouver au niveau des cônes, des P et PM. La présence, dans les $P$ et PM des nonmammaliens, de molécules apparentées à l'opsine [10], à l' $\alpha$ transducine [11] et à l'antigène$\mathrm{S}[10,12]$ permet, avec d'autres données biochimiques, de considérer que les mécanismes de la phototransduction sont voisins dans la pinéale et dans la rétine. En revanche, on ignore si dans les $\mathrm{Pi}$ des mammiferes l'existence des molécules apparentées à l'antigène $\mathrm{S}$ et à l'opsine, et d'une activité rhodopsine-kinase [13] est significative d'une photosensibilité directe résiduelle, ou d'homologies entre des molécules impliquées dans la phototransduction et l'adréno-transduction (exemple : entre récepteur $\beta$-adrénergique et opsine).

Protéines de liaison des rétinoïdes. La photolyse de la rhodopsine, aboutissant à la dissociation de l'opsine et du tout-trans rétinal, est suivie de la régénération du complexe opsine-11-cis rétinal. Les réactions enzymatiques impliquées dans la transformation du rétinal se produisent dans des cellules de la rétine et dans celles de l'épithélium pigmentaire. Le transfert des rétinoïdes au sein de ces cellules, ou entre les deux tissus qu'elles constituent, s'opère par l'intermédiaire de protéines de liaison. Parmi celle-ci, l'interstitial retinoid-binding protein (IRBP), une glycoprotéine de 144 kD synthétisée par les PR, assure le transfert de rétinoïdes dans l'interstice situé entre les deux tissus. Une protéine possédant l'immunoréactivité de I'IRBP (dont la fonction n'est pas établie) est présente dans les PM de la caille et dans la pinéale de bœuf (10].

Protéines de liaison du $\mathrm{Ca}^{++}$. Au cours (a) de la photo- ou de l'adréno-transduction (voir cidessous), (b) de la neurotransmission et (c) de l'induction de la synthèse protéique, le rôle des ions $\mathrm{Ca}^{2+}$ est étudié dans les PR ou dans les Pi. Une protéine de liaison $\mathrm{du} \mathrm{Ca}^{2+}$, la calbindine-27 kD (non spécifique des phototransducteurs), vient d'être découverte dans les cônes rétiniens, les $\mathrm{P}$, les PM et les Pi [14].

\section{Les molécules " messages"}

Les PR, P, PM et Pi élaborent plusieurs types de molécules "messages".

Neurotransmetteurs. La dépolarisation (à l'obscurité) et l'hyperpolarisation (à la lumière) sont communes aux PR et $\mathrm{P}[2,3,8$, $9,15]$. La libération (dépendante du $\mathrm{Ca}^{2+}$ ) d'un neurotransmetteur excitateur au pôle synaptique, est liée aux états de dépolarisation ou d'hyperpolarisation des PR et des $P$, et est responsable de réponses quasi immédiates des neurones situés en aval. L'aspartate ou le glutamate sont considérés comme les neurotransmetteurs des PR et vraisemblablement des $\mathrm{P}[2,3$, 15]. Au niveau des pôles synaptiques, il existe non seulement des analogies structurales mais aussi fonctionnelles. Ainsi, l'aspartate et le glutamate ont des effets soit dépolarisants sur une classe de cellules bipolaires et horizontales rétiniennes et sur les neurones de la pinéale, soit hyperpolarisants sur une autre classe de cellules bipolaires rétiniennes $[2,3,7,15]$. Le traitement de ces messages s'effectue localement, selon des mécanismes complexes, dans la rétine. A partir de cette dernière, ou de la pinéale des nonmammaliens, les informations sont al. $\alpha$-Transducin immunoreactivity in retinae and sensory pineal organs of adult vertebrates. Proc Natl Acad Sci USA 1986 ; 83 : 912-6. 


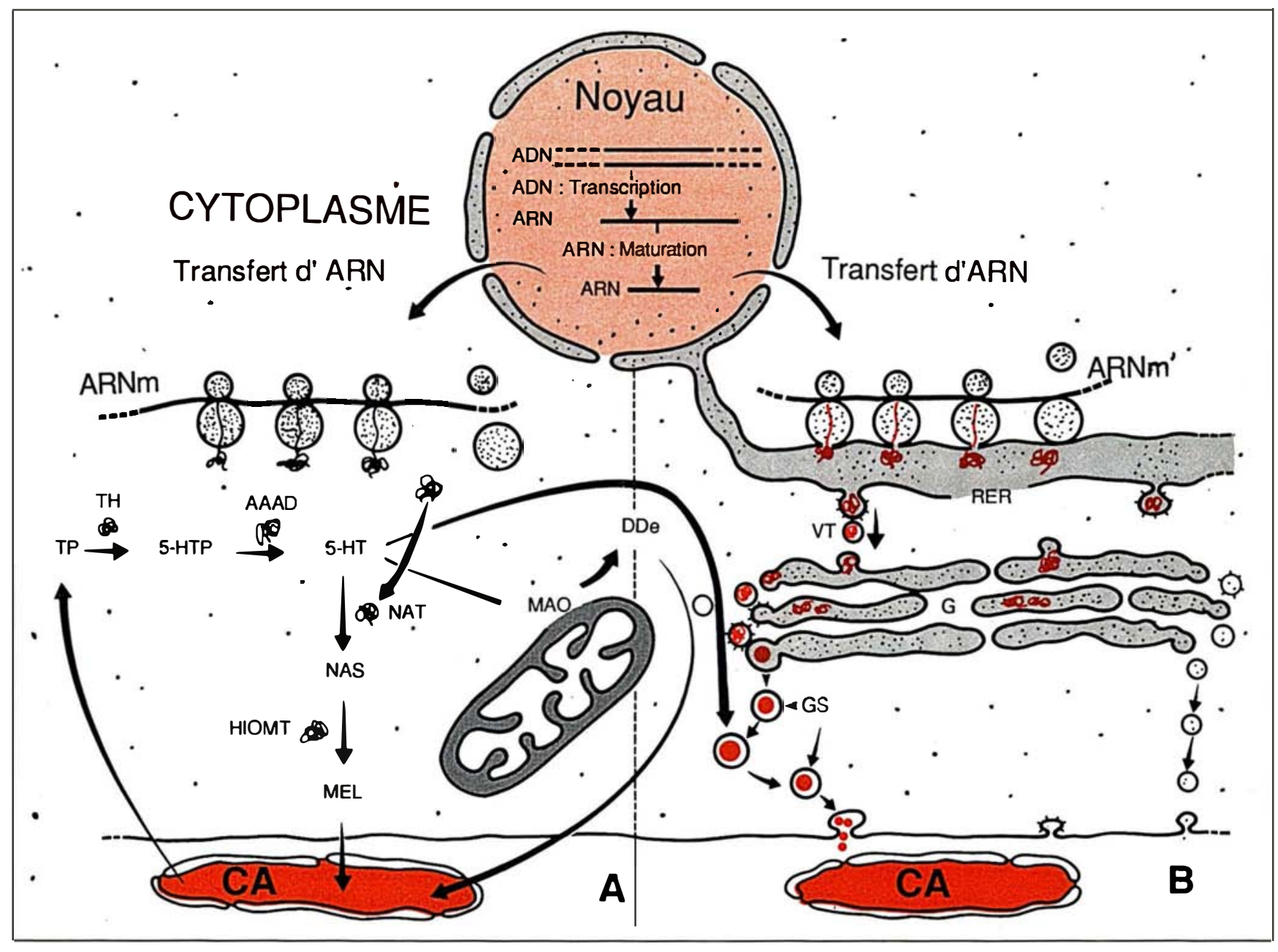

Figure 2. Processus sécrétoires dans les transducteurs pinéaliens (abréviations : voir figure 1). A. Métabolisme indolique (simplifié) dans le cytosol. Les polysomes synthétisent les enzymes impliquées dans la production de mélatonine (MEL). Au cours de la synthèse de mélatonine, à partir du tryptophane (TP), quatre enzymes interviennent successivement $I T H=$ tryptophane-hydroxylase; $A A A D=$ amino-acide aromatique-décarboxylase; NAT = Nacétyltransférase ; HIOMT = hydroxy-indole-O-méthyltransférase). 5-HTP = 5-hydroxytryptophane; 5-HT = sérotonine; NAS = N-acétylsérotonine; MEL = mélatonine; DDe = dérivés désaminés par la monoamine-oxydase (MAO). B. Sécrétion protéique (neuropeptide(s) hypothétique(s)) par le système endomembranaire. RER = réticulum endoplasmique rugueux; $V T=$ vésicules de transition; $G=$ dictyosome; $G S=$ grains de sécrétion.

ensuite acheminées, par plusieurs voies neuronales, à différents étages du système nerveux central $[2$, 6] où elles sont décodées et impliquées dans des fonctions variées (voir introduction; [6]). La voie rétino-hypothalamique retiendra particulièrement notre attention : elle transmet l'information photopériodique à des oscillateurs circadiens.

Mélatonine (MEL). Elle fut découverte en 1958 dans la pinéale de bœuf [16]. Ultérieurement, il a été établi que sa synthèse s'effectue dans la pinéale et dans la rétine d'espèces appar$\mathrm{m} / \mathrm{s} n^{\circ} 1$ vol. 4, janvier 88 tenant à différentes classes de vertébrés. La pinéale dispose de l'équipement enzymatique (figure $2 A)$ lui permettant de synthétiser, à partir du tryptophane, la sérotonine et ses dérivés 5-méthoxyindoliques dont la MEL. Parmi les voies du métabolisme indolique, la production de MEL a retenu une attention particulière (figure 2A). Dans la pinéale et dans la rétine, la MEL est élaborée en deux étapes à partir de la sérotonine : la première (sérotonine $\rightarrow \mathrm{N}$-acétylsérotonine) fait intervenir la sérotonine-Nacétyltransférase (NAT) en pré- sence d'acétyl-CoA et la seconde (N-acétylsérotonine $\rightarrow$ MEL) l'hydroxyindole-O-méthyltransférase (HIOMT), en présence de Sadénosyl-méthionine [10,17]. Les transducteurs de la pinéale sont responsables du métabolisme indolique $[5,6,15,18]$. Des travaux préliminaires plaident en faveur de la synthèse de la MEL dans les PR de mammifères [10]. Les incidences de la synthèse nocturne de la MEL sont évoquées ultérieurement.

Sécrétion protéique. Dans les $\mathrm{P}$, $\mathrm{PM}$ et $\mathrm{Pi}$, des grains de sécrétion (diamètre moyen : $100 \mathrm{~nm}$ ) d'ori- 
gine golgienne (figures 1 et 2B) ont une matrice riche en protéines, suggérant l'existence de molécules " messages " de type peptidique dont la structure et le rôle sont à déterminer [19]. De tels grains de sécrétion ont parfois été signalés dans les PR.

\section{L'activité rythmique journalière}

Des recherches effectuées à l'échelle cellulaire et moléculaire ont révélé une activité rythmique des PR, P, PM et Pi. La période de $24 \mathrm{~h}$ du rythme de cette activité est calibrée par la photopériode qui est un donneur de temps (Zeitgeber) ou synchroniseur. Pour une variable donnée (exemple : la MEL plasmatique), le chronogramme du rythme est établi à partir de valeurs moyennes, obtenues à intervalles réguliers au cours d'un ou de plusieurs nycthémères. Ces valeurs permettent de tracer une courbe caractérisée par sa période (par exemple : $24 \mathrm{~h}$ ), la situation de son pic (acrophase), son amplitude (différence pic-creux) et son niveau moyen par $24 \mathrm{~h}$.

Les rythmes nycthéméraux de la production de MEL et du renouvellement des disques des photorécepteurs sont les plus étudiés. Quels que soient les comportements (nocturne ou diurne) ou l'habitat des vertébrés étudiés, les pics d'activité NAT et de concentration de la MEL dans la pinéale et dans la rétine, sont observés à l'obscurité $[10,15,20,21]$ (figure $3 B-D)$. Le taux de MEL plasmatique montre des variations similaires, y compris chez l'homme (voir ultérieurement). Ce sont les fluctuations de l'activité NAT (figure $3 B-D$ ) qui engendrent les changements de concentrations de la MEL pinéalienne, rétinienne et plasmatique.

Chez des mammiferres et autres vertébrés, la durée de synthèse nocturne de la MEL est fonction de la longueur de la phase nocturne $[10,22,23,24]$ (figure $3 B$ $D)$. De plus, au cours de cette phase, la lumière peut inhiber telle inhibition dépend des paramètres physiques du stimulus (voir introduction), dont les valeurs efficaces different selon les espèces, voire chez une même espèce en fonction des caractéristiques de la lumière perçue antérieurement [10, 23, 25]. En bref, si le chronogramme se rapportant à la synthèse de la $\mathrm{MEL}$ a une allure sinusoïdale, celle-ci varie dans le détail d'un cycle de $24 \mathrm{~h}$ à l'autre et peut traduire fidèlement - au moins chez les espèces dites " photosensibles" - les variations du stimulus lumineux. Ainsi, la MEL, dont la concentration reflète, au sein de différents organismes, les variations quotidiennes de l'éclairement, est qualifiée de "messager de la photopériode".

$\mathrm{Au}$ cours du cycle de $24 \mathrm{~h}$, les $\mathrm{PR}$ renouvellent un certain contingent de leurs disques et les photopigments transmembranaires correspondants. Les paquets de disques sont éliminés à la partie distale des segments externes, au cours de la séquence diurne (bâtonnets) ou nocturne (cônes) [10]. Cette disparition est compensée par la genèse de nouveaux disques à la partie proximale des segments externes. L'étude du renouvellement des disques du segment externe des $P$ et PM demeure incomplète.

D'autres variations journalières ont été décrites [23] dans les PR et/ou $\mathrm{P}, \mathrm{PM}$ et $\mathrm{Pi}_{1}$ : volume du cytoplasme et du noyau, volume et surface membranaire du réticulum endoplasmique et des dictyosomes, nombre de grains de sécrétion, nombre et longueur des rubans synaptiques (figure $3 H-L$ ). Des variations nycthémérales de concentrations d'ARNm, de l'activité rhodopsine kinase, de concentrations de phospholipides, de GMPc, de sensibilité de récepteurs membranaires, etc., ont également été établies.

\section{Oscillateurs circadiens et transduction}

$\mathrm{Au}$ cours du nycthémère, l'alternance lumière/obscurité ou $\mathrm{L} / \mathrm{D}$ $(\mathrm{L}=$ light $; \mathrm{D}=$ dark) gouverne directement au niveau des PR et au moins des $P$ un rythme de $24 \mathrm{~h}$ d'hyperpolarisation/dépolarisation. Néanmoins, pour une majorité des rythmes énumérés cidessus, des mécanismes de contrôle plus complexes sont mis en jeu. Ceux-ci ont été décryptés en choisissant principalement comme paramètre les fluctuations de la synthèse de la MEL dans la glande pinéale.

Pinéale. Lorsque des rats ou des poulets sont placés dans des conditions d'isolement temporel (D/D ou $L / L$ ), le rythme de synthèse de la MEL évolue en " libre cours "; la hauteur du pic nocturne de l'indole se trouve réduite en $\mathrm{D} / \mathrm{D}$ (figure $3 F$ ) et très réduite en $\mathrm{L} / \mathrm{L}$ figure $3 G)[10,22]$. Le rythme, ici engendré par des oscillateurs internes, est dit circadien (circa : environ; dies : jour) et endogène. Lorsque les animaux sont exposés à l'alternance $\mathrm{L} / \mathrm{D}$ du cycle de $24 \mathrm{~h}$, la photopériode (et éventuellement d'autres synchroniseurs environnementaux) impose(nt) aux oscillateurs circadiens une période de $24 \mathrm{~h}$. Ces oscillateurs montrent une grande souplesse fonctionnelle : après inversion (D/L à la place de L/D) ou glissement de phases, l'acrophase de la MEL se déplace jusqu'à ce qu'elle retrouve, au bout de quelques jours, sa position "nocturne " initiale [23].

Chez les mammiferes, les mécanismes de contrôle de la synthèse de la MEL dans la pinéale sont principalement étudiés chez le rat $[17,21,22]$. Chez l'homme, les mécanismes de régulation sont voisins. Via les photorécepteurs rétiniens et une projection directe rétino-hypothalamique, la photopériode entraîne les oscillateurs circadiens, localisés dans les noyaux suprachiasmatiques de l'hypothalamus. Les signaux des oscillateurs sont acheminés par des chaînes neuronales (figure 4A), empruntant le tronc cérébral puis la voie orthosympathique aboutissant à proximité des $\mathrm{Pi}$. C'est la noradrénaline, libérée de nuit par les fibres orthosympathiques qui, via le système récepteur $\beta_{1}$-adrénergique-adénylcyclase des $\mathrm{Pi}$, 


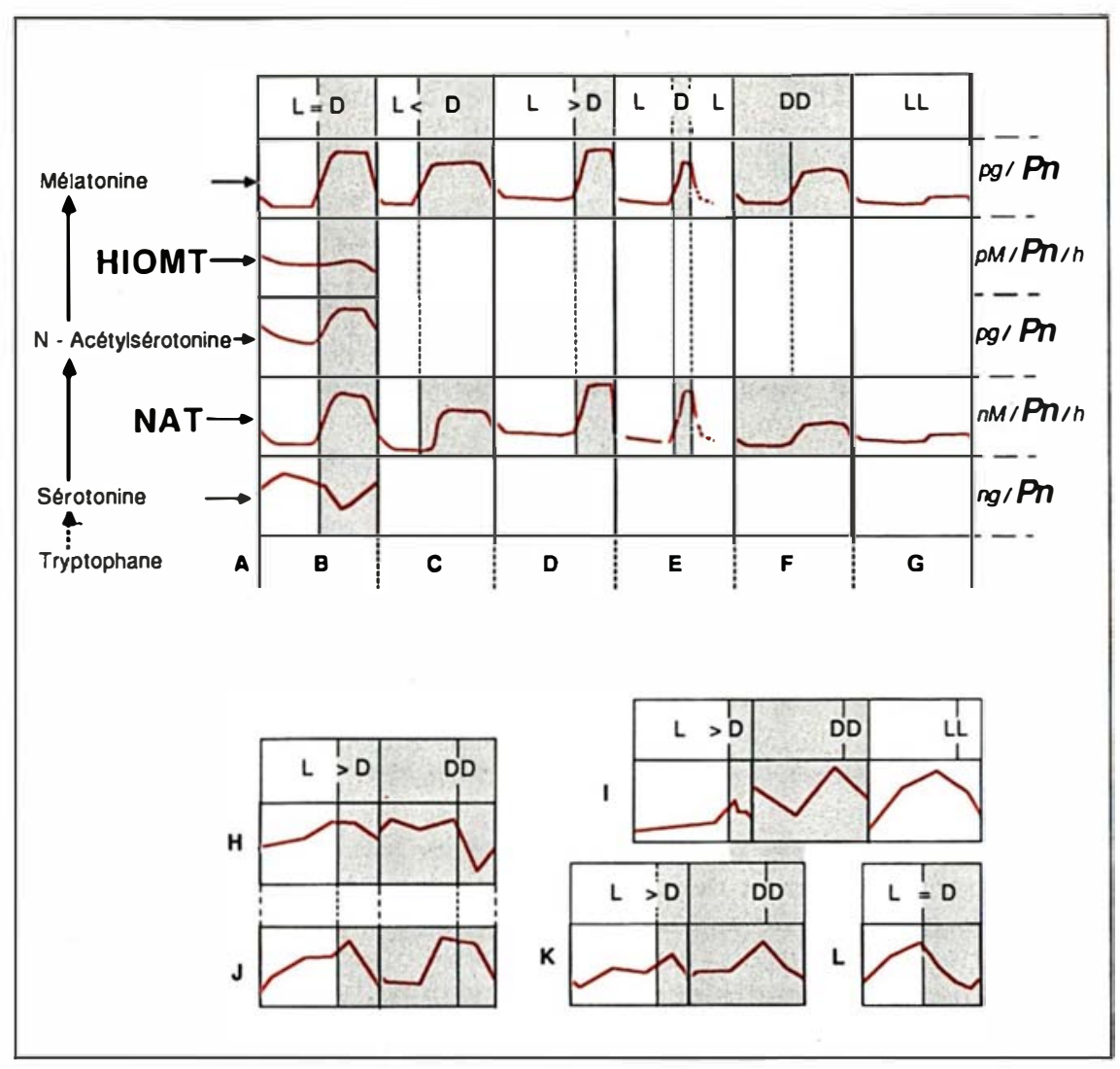

Figure 3. Pinéalo : variations circadiennes du métabolisme indolique (AG) ot de constituants cellulaires (H-L) dans différentes conditions photopériodiques (abréviations : voir figure 2). A. Biosynthèse de la mélatonine. B. Rythmes circadiens des indoles et des enzymes du métabolisme indolique chez la plupart des vertébrés. Photopériode : $L(12 h)=D(12 h)$. C-G. Chronogrammes des concentrations de la mélatonine et de l'activité de la NAT (chez les homéothermes) dans différentes conditions photopériodiques (voir en haut de la figurel. Les unités de concentrations d'indoles et d'activités enzymatiques sont rapportées par pinéale (Pn), à droite du schéma. H-K. Dans les $P$ de la pinéale du poisson rouge : variations du volume du noyau $\left(\mu \mathrm{m}^{3}\right)(\boldsymbol{H}), d u$ nombre de rubans synaptiques par unité de surface (I), de la surface $\left(\mu \mathrm{m}^{2}\right)$ du réticulum endoplasmique rugueux (J), et du volume de l'appareil de Golgi (K). L. Dans les prolongements des Pi du lapin, variations du nombre de grains de sécrétion par unité de surface.

induit l'activation de la NAT et, en conséquence, la synthèse nocturne de la MEL $(17,21,22]$ (figure $4 A$ ). De plus, cette synthèse est modulée par des hormones et par les neuromédiateurs de fibres nerveuses, en provenance du système nerveux central.

Chez les oiseaux, la régulation de la synthèse de la MEL dans la pinéale, étudiée chez le poulet, fait appel à des mécanismes plus complexes. La pinéale contient une population d'oscillateurs circadiens - dont chacun pourrait $m / s n^{\circ} 1$ vol. 4, janvier 88 la pinéale, contrôlant la production de MEL (inhibition de jour ; synthèse de nuit) ;

- d'autre part, la photopériode synchronise des oscillateurs circadiens externes à la pinéale (probablement localisés dans les noyaux suprachiasmatiques [27]) dont les signaux empruntent la voie orthosympathique se terminant à proximité des PM (figure $4 B)$. La noradrénaline, libérée de jour par les fibres orthosympathiques [27], contribue à inhiber l'activité NAT, via des récepteurs $\alpha$ post-synaptiques, appartenant selon toute vraisemblance aux PM ([28] et en préparation) (figure $4 B)$. Cette libération diurne de la noradrénaline semble correspondre à un signal complémentaire de synchronisation des oscillateurs circadiens intrapinéaliens.

Un oscillateur circadien interne à la pinéale a également été mis en évidence chez un lézard (anole) et chez le brochet ([29] et Falcón et al., en préparation) (figure $4 C$ ). Chez l'anole, à l'inverse du poulet, l'oscillateur est auto-entretenu. Rétine. Les mécanismes responsables de la rythmicité de la synthèse de la MEL et du renouvellement des disques des segments externes dépendent aussi d'oscillateurs circadiens. Un oscillateur interne à la rétine (xénope) maintient au moins pendant 3 jours le rythme circadien de l'activité NAT (figure 4D). De plus, la dopamine libérée au cours de la phase diurne par les cellules amacrines rétiniennes inhibe l'activité NAT, via des récepteurs $\mathrm{D}_{2}$ (figure 4D) [10]. Néanmoins, sur ce modèle, l'intervention d'oscillateurs du système nerveux central, les relations éventuelles entre oscillateurs circadiens intrarétiniens et neurones dopaminergiques, ainsi que les sites de synthèse de la MEL (vraisemblablement dans les PR) doivent être encore précisés.

Un thème commun. Dans la pinéale et la rétine des vertébrés, la synthèse nocturne de la MEL et l'activité rythmique des phototransducteurs [23] sont contrôlées par des oscillateurs circadiens synchronisés, selon une période de 


\section{REFERENCES}

12. Collin JP, Mirshahi $M$, Briseon $P, F a l-$ đon J, Guerlotté J, Faure JP. Pineal-recinal molecular relarionships : distribution of a $S$ antigen $*$ in the pineal complex. Naurosciorce $1986 ; 2: 657-66$.

13. Ho AK, Somers RL, Klein DC. Development and rogulanion of hodopsin kinase in rat pineal and retina. $J$ Nawochem 1986 ; 46 ; 1176-9.

14. Roman A, Brisson P, Pasteels B, Demol S, Pochet R, Collin JP. Pineal-retinal molecular relationships ; immunocytochemical evidence of calbindin-27 kDa in pineal transducers. Brain Ras 1987 (sous presse).

15. Collin JP, Meissl H, Voisin P, Brisson P, Falcón J. Rhythmic signals of pineal transducers : physiological, biochemical and cytochemical evidence. In : Reiter RJ, Karasek M, eds. Adoances in Pineal Research : 1. London : John Libbey and Co Ltd, 1986 : 41-50.

16. Lemer AB, Case JD, Takakashi Y, Lee Th, Mori W. Isolanion of melatonin, the pineal gland factor that lightens melanocytes. $\mathrm{J} \mathrm{Am}$ Chem Sac $1958 ; 80$ : 2587.

17. Evred D, Klark S. Photoperiodism, Mela tonin and the Pineal (Ciba Foundation Symposium, 117). London: Pitman, 1985 : 1-333.

18. Collin JP. Cellular biology of the pineal organ with special reference to some known and other hypothetical messenger substances ; the CRL concept and outlook. In : Mess B a al., eds. The Pinoal Gland: Current State of Pineal Research. Budapest : Akadémiai Kiad6, 1985 : 91-110.

19. Collin JP. New data and vistas on the mechanisms of secretion of proteins and indoles in the mammalian pinealocyte and its phylogenetic precursors ; the pinealin hypothesis and preliminary comments on membrane traffic. In : Okeche A, Pévet P, eds. The Pineal Organ : Photobiology-Biochronometry-Endocrinology. Amsterdam : Elsevier/North-Holland Biomedical Press, 1981 : 187-210.

20. Falcón J, Guerlotté JF, Voisin P, Collin JP. Rhythmic melatonin biosynthesis in a photoreceptive pineal organ : a study in the pike Neuroendocrinology $1987 ; 47$ : 479-86.

21. Reiter RJ. Pineal indoles : production secretion and actions. In : Müller EE, Mac Leod RM, eds. Nawoendocrine Perspectives, Vol. 3. Amsterdam : Elsevier Science Publishers, 1984 : 345-77.

22. Binkley SA. Circadian rhythms of pineal functions in rats. Endacr Rev $1983 ; 4: 255-70$.

23. Collin JP, Voisin P, Falcón J, Brisson $\mathbf{P}$. Evolution and environmental control of secretory processes in pineal transducers. In : Scharrer B, Hartwig HG, Korf HW, eds. Functional Morphology of Neuroendocrine Systems Evolutionary and Environmental Aspects. Berlin :
$24 \mathrm{~h}$, par un puissant Zeitgeber : le cycle photopériodique $\mathrm{L} / \mathrm{D}$. Le rythme de la MEL, au moins dans la pinéale, semble être également influencé par d'autres synchroniseurs qui doivent être clairement définis $[23,24]$. Ainsi, le cycle thernique de l'environnement est un important facteur d'entraînement chez les poikilothermes [23]. La pluralité des systèmes de régulation impliqués dans la synthèse de la MEL pourrait être liée à l'évolution des vertébrés et à leur adaptation aux variations périodiques du milieu.

\section{La mélatonine, synchroniseur de rythmes}

Rappelons que les oscillateurs circadiens, entraînés par les facteurs environnementaux, sont non seulement générateurs du rythme de la MEL mais également des oscillations circadiennes de nombreuses fonctions (exemple : régulations neuroendocriniennes et comportementales). Dans quelle mesure la MEL, vecteur de l'information photopériodique, permet-elle aux mammiferes de vivre en harmonie avec leur environnement? Existe-t-il des relations entre les oscillations circadiennes et circannuelles de l'indole et celles de diverses fonctions? Quelques exemples montrent que la MEL de la pinéale et de la rétine est - via ses actions neuroendocrines, paracrines et autocrines - un synchroniseur interne. Libérée dans l'effluent sanguin par la pinéale (et également par la rétine de nonmammaliens), cette hormone se rencontre aussi dans le liquide céphalo-rachidien.

Synchronisation des rythmes circadiens. Contrairement à celle des non-mammaliens, la pinéale des mammiferes ne semble pas pouvoir engendrer la rythmicité de fonctions et de métabolismes [10]. Les travaux préliminaires chez le rat, suggérant l'implication de la pinéale dans la synchronisation de rythmes comportementaux [30], ont pu être précisés. Ainsi après : (a) désynchronisation externe (les rythmes évoluent en "libre cours "), ou (b) désynchronisation interne (rythmes à périodes différentes à cause du découplage des oscillateurs ; arythmie...) ou (c) glissement de phase, l'administration appropriée de MEL corrige les altérations des rythmes [30]. L'hypothèse selon laquelle la MEL contrôle, par rétroaction, les oscillateurs responsables de l'induction de sa sécrétion, est plausible. En effet, la lésion des noyaux suprachiasmatiques exclut toute resynchronisation [30] ; chez le rat, les noyaux suprachiasmatiques et l'éminence médiane correspondent aux cibles hypothalamiques de la MEL (Vanecek et al., en préparation). Dans la rétine, la MEL a des actions autocrines (contrôle circadien du renouvellement des disques ou des processus contractiles, dans les PR) et paracrines (inhibition circadienne de la libération de la dopamine des cellules amacrines, contrôle circadien de la migration des pigments de mélanine dans l'épithélium pigmentaire) [10]. Des récepteurs mélatoninergiques sont reconnus au niveau des cellules amacrines rétiniennes [10].

Synchronisation des rythmes circannuels. Les mammiferes vivant dans les régions tempérées doivent faire face aux fluctuations saisonnières du milieu. Pour que la naissance et la croissance de leur progéniture puissent s'effectuer à l'époque la plus propice de l'année, la nécessité de prévoir et de préparer les conditions de survie s'impose à chaque espèce. Chez de nombreux animaux, la sensibilité à l'éclairement quotidien constitue un repère majeur pour la mise en place des stratégies adaptatives. Selon des mécanismes encore imparfaitement connus, le système circadien des mammiferes mesure la durée de l'éclairement et l'interprète pour stimuler ou inhiber différents systèmes de régulation. Ainsi au cours de l'année, certaines espèces mettent à profit la durée décroissante, d'autres la durée croissante de l'éclairement quotidien. La durée respectivement croissante ou décroissante de la 


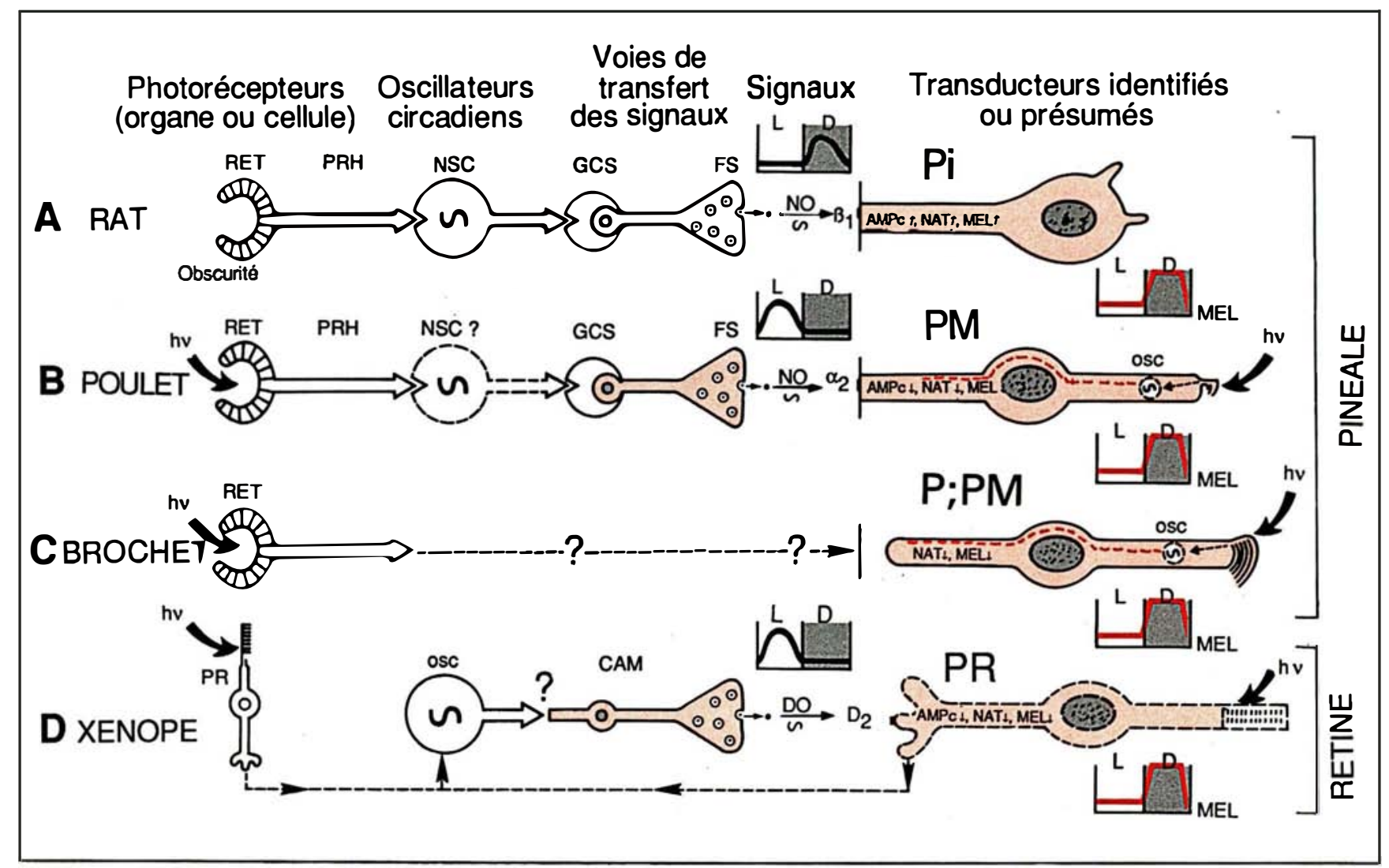

Figure 4. Systèmes de régulation du rythme circadien de la synthèse de mélatonine dans les transducteurs pinéaliens (A-C) ot les photorécepteurs rétiniens (D). Les chronogrammes correspondent aux fluctuations des signaux (NO = noradrénaline ; $D O=$ dopamine) et aux variations circadiennes des concentrations de mélatonine (MEL). A. Chez le rat (voir texte), sont représentés de gauche à droite, la rétine (RET), la projection rétino-hypothalamique (PRH), les noyaux suprachiasmatiques (NSC), les ganglions cervicaux supérieurs (GCS), les terminaisons orthosympathiques (FS) libérant la noradrénaline (NO) et les pinéalocytes (Pi) avec les récepteurs $\beta_{1}$-adrénergiques $\left(\beta_{1}\right)$. B. Chez le poulet (voir texte; mêmes légendes que chez le rat). La localisation de l'un des nombreux oscillateurs (OSC) intrapinéaliens est hypothétique. C. Chez le brochet, un système de régulation extrapinéalien est inconnu. D. Rétine du xénope (voir texte; cellules amacrines = CAM). Les flèches, sur les traits en pointillés, désignent l'entraînement de l'oscilla-

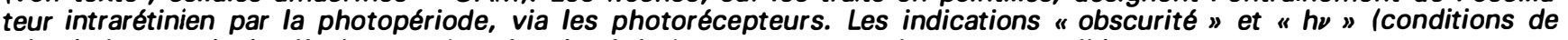
stimulation par la lumière) sont données à côté des structures qui y sont sensibles.

sécrétion nocturne de la mélatonine $[24,30]$ peut être "lue " par des régions spécialisées du cerveau qui contrôlent les mécanismes impliqués dans les régulations neuroendocriniennes (dont l'axe hypothal a mo-h y po physogonadique) et comportementales. Ainsi, via la MEL, les $\mathrm{Pi}$ et les structures centrales, situées en amont et en aval, orchestrent les cycles biologiques annuels de la reproduction, des mues du pelage, du métabolisme énergétique [17, $21,24,30-32$ ]. Notons que la pinéalectomie inhibe les changements induits par la photopériode. L'ensemble de ces observations a conduit récemment à des applications dans le domaine de l'amé- lioration des productions animales. En résumé, par les actions locales ou à distance de la MEL, les phototransducteurs diencéphaliques contribuent à l'optimisation des relations de l'organisme avec son environnement.

\section{Perspectives médicales}

Les données récentes portant sur la MEL et sur les protéines de la phototransduction débouchent sur des perspectives médicales.

Mélatonine humaine. L'essor sans précédent des recherches sur la MEL humaine est lié aux acquis chez l'animal, et à la fiabilité des techniques de dosages du 5-méthoxyindole ou de ses métabolites [24, 30, 33, 34]. Chez l'homme, les dosages effectués au cours du nycthémère, à partir du plasma, de l'urine et de la salive, servent d'index de l'activité circadienne de la pinéale $[30,33]$. Après pinéalomectomie, dans les cas de néoplasmes des $\mathrm{Pi}$, la $\mathrm{MEL}$ plasmatique est indétectable, ce qui suggère son origine à partir de la pinéale exclusivement [ 30 , 33]. L'existence (a) d'hydroxyindole-O-méthyltransférase (HIOMT) dans les $\mathrm{Pi}$, (b) d'une activité NAT accrue de nuit dans la pinéale (examens post-mortem) et (c) de variations très marquées du taux de MEL plasmatique (non détectable de jour ; plusieurs pics pendant la nuit), plaide en 
faveur d'une sécrétion circadienne de l'indole par les Pi humains [10, $24,30,33]$. Des variations de cette sécrétion soit interindividuelles journalières, soit saisonnières (maximale en hiver et en été ; minimale au printemps et en automne), ont été établies [24, 30, 33]. De plus, le taux nocturne de la MEL, faible au cours de la première année, atteint un maximum entre 1 et 3 ar:s, puis diminue progressivement jusqu'à la puberté, pour se stabiliser à l'âge adulte et décroître au cours de la vieillesse. Des observations principalement cliniques, plaident en faveur de l'existence de mécanismes de régulation de la synthèse de la MEL, voisins de ceux décrits chez le rat (figures $4 A$ et 5 ). La photopériode constitue chez l'homme l'un des synchroniseurs des oscillateurs. L'inhibition de la synthèse nocturne de la MEL nécessite néanmoins une intensité lumineuse (1 500 lux) très supérieure à celle qui, chez les animaux, est efficace; 500 lux suffisent chez des patients atteints de psychose maniaco-dépressive [30]. Les relations entre désynchronisations, MEL et troubles ou affections variés sont à l'étude [24, 30, $33,34]$. Des résultats préliminaires montrent que la MEL réduit les troubles du décalage horaire occasionnés par les vols transméridiens (diminution du temps de latence du sommeil et amélioration de sa qualité ; accélération de la resynchronisation des rythmes circadiens de la MEL et du cortisol) [34]. L'optimisation des conditions du travail posté fait également l'objet de recherches [30].

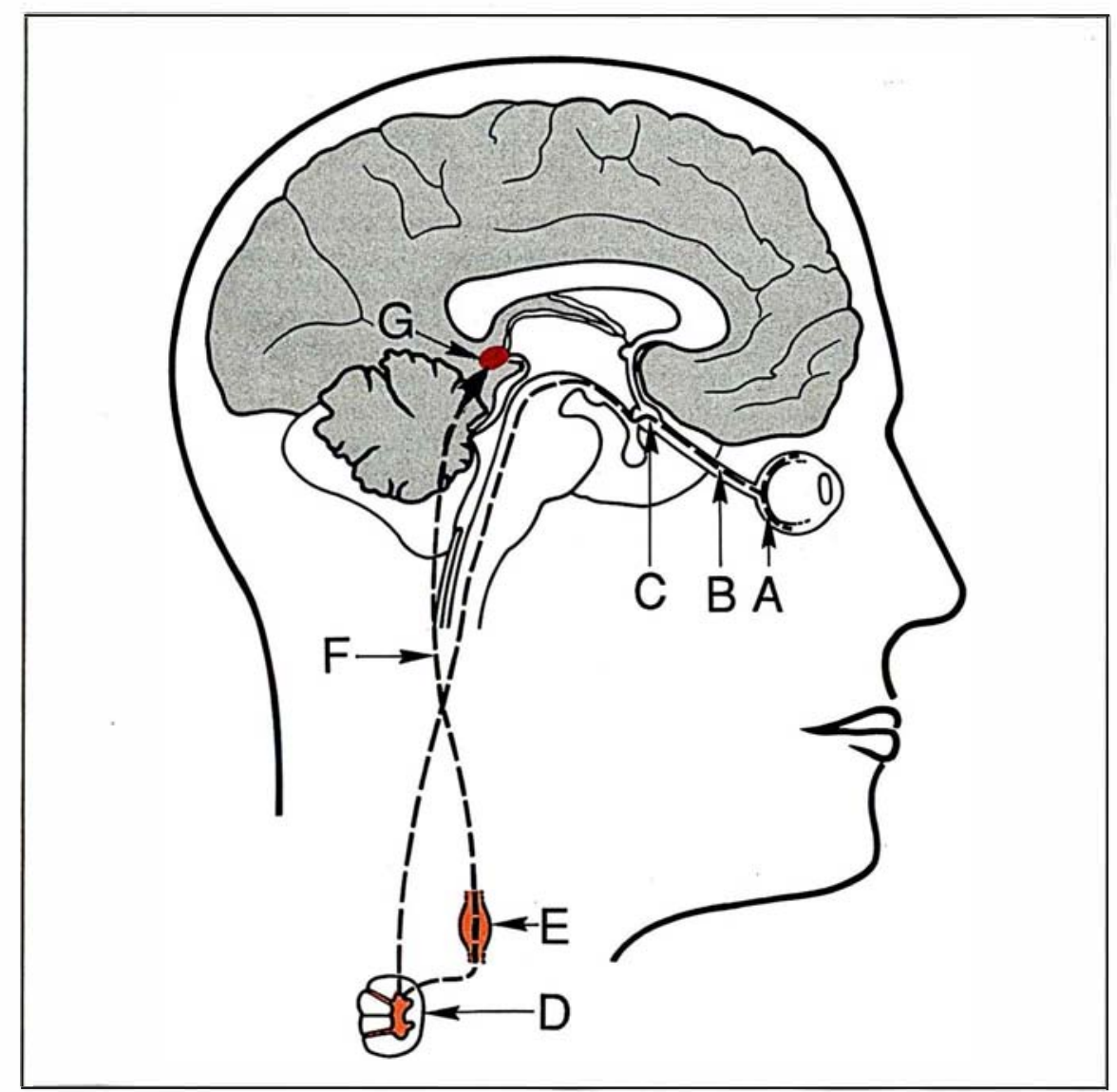

Figure 5. Voies nerveuses impliquées dans la régulation de la synthèse de la mélatonine de la pinéale humaine. $A=$ rétine; $B=$ nerf optique ; $C=$ hypothalamus (avec chiasma optique, noyaux suprachiasmatiques, etc.) ; $D=$ moelle épinière $; E=$ ganglions cervicaux supérieurs; $F=$ fibres
La MEL a des effets hypnotiques et modifie le timing de la fatigue $[24,34]$; elle pourrait participer à la régulation physiologique du sommeil, en déterminant la phase de son rythme et celle du rythme de la somnolence [30]. Le dysfonctionnement de l'organisation temporelle s'observe dans des cas d'affections neuro-psychiatriques, dont certaines à manif estation cyclique $[17,30,33,35]$. Chez les sujets atteints de dépression endogène, les rythmes circadiens (sommeil, température corporelle, hormones...) montrent des altérations d'amplitude (exemples : hyposécrétion de mélatonine et hypercortisolémie) et de phase [33, 35]. Des variations saisonnières, hypo- ou hypersécrétion de la MEL, sont également observées dans certains états dépressifs. L'amélioration clinique de la dépression s'oriente vers le recouplage oscillateurs/environnement (la chrono- et la photothérapie demeurent des traitements expérimentaux) et la recherche de molécules à vertus synchronisantes [35]. Dans l'algie vasculaire de la face, une hyposécrétion de MEL est reconnue, ainsi qu'une avance de phase de son rythme; une avance de phase affecte également le rythme du cortisol [33]. Chez les schizophrènes, les taux nocturnes de MEL sont significativement réduits [33].

Les observations, chez les patients dépressifs ou atteints de la maladie de Cushing, ont suggéré des relations fonctionnelles étroites entre pinéale et axe hypothalamohypophyso-surrénalien [30, 33]. $L$ 'influence que pourrait exercer la pinéale sur la fonction de reproduction n'est pas établie ; des investigations visent à montrer des relations entre, par exemple, MEL et cycle menstruel ou MEL et puberté $[30,33]$. Enfin, c'est dans une perspective chronobiologique que se développent des recherches relatives à l'influence de la MEL sur des néoplasies et sur le système immunitaire.

La synthèse de MEL dans les photorécepteurs rétiniens humains est suggérée par la présence de l'HIOMT [10], mais son action $\mathrm{m} / \mathrm{s} n^{\circ} 1 \mathrm{vol}$. 4, janvier 88 

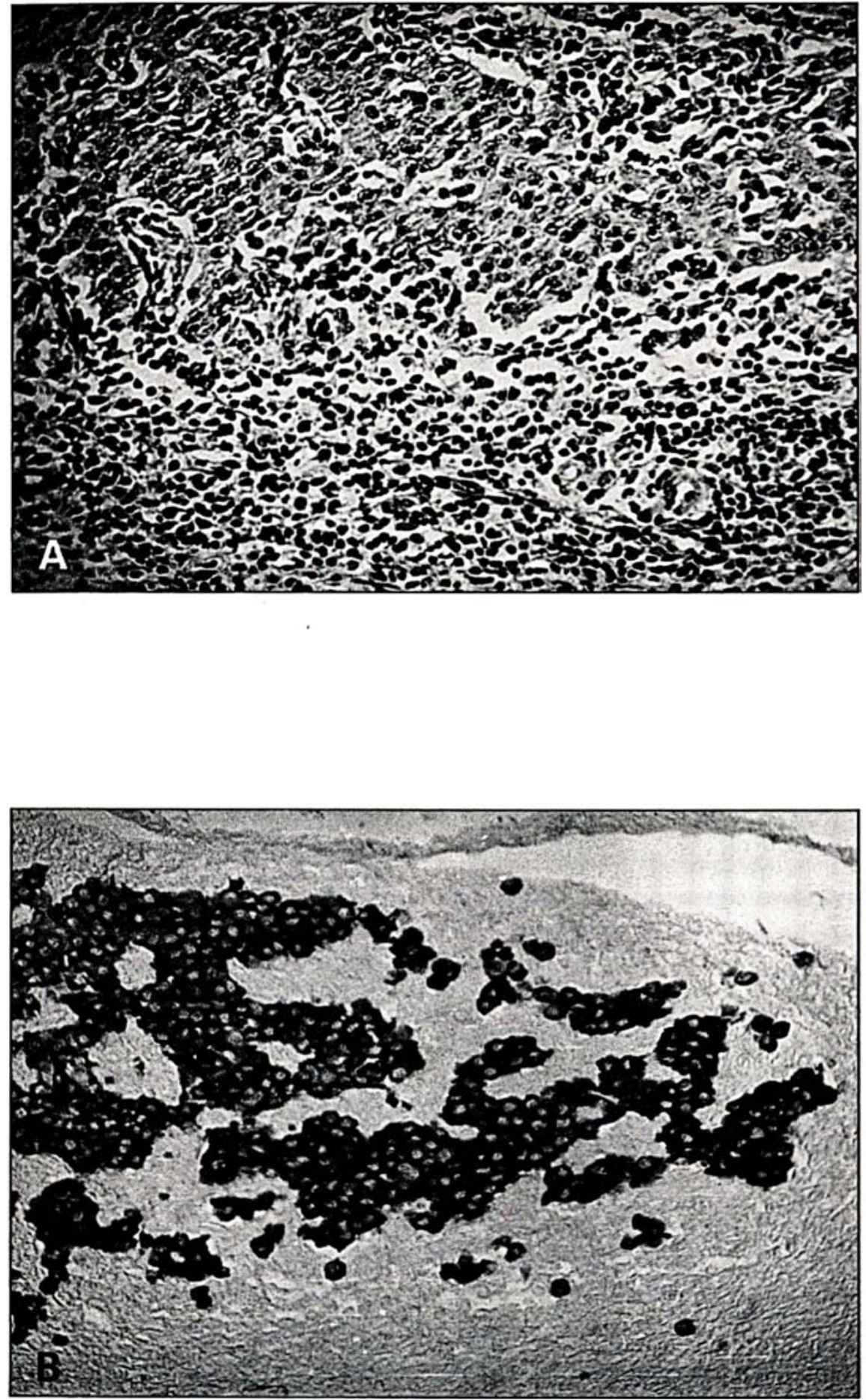

Figure 6. Pinéalite auto-immune chez un cobaye, 41 jours aprés liimmunisation avec un homogénat de sa propre rétine isolée de l'un des yeux énucléé, injecté dans le coussinet plantaire avec adjuvant de Freund. A. L'infiltrat lymphocytaire est très dense dans la partie périphérique de la pinéale et moins dense en son centre (hématoxyline-éosine). B. Sur une coupe voisine, l'ampleur de la destruction des pinéalocytes est illustrée ; l'immunoréactivité pour l'antigène-S n'est observée que dans les pinéalocytes encore sains (technique biotine-streptavidine-peroxydase avec un anticorps monoclonal contre l'antigène-S).

$\mathrm{m} / \mathrm{s} n^{\circ} 1$ vol. 4, janvier 88 locale éventuelle, suivie de sa dégradation, ne sont pas établies. Maladies auto-immunes. Une propriété remarquable de protéines de la phototransduction, communes à la rétine et à la pinéale, est leur auto-antigénicité. L'immunisation de certains mammiferes avec l'antigène-S, l'IRBP ou la rhodopsine extraits de la rétine provoque l'uvéorétinite autoimmune expérimentale (UAE), maladie inflammatoire de l'œil, modèle pour l'étude des uvéorétinites humaines [36]. Or, l'UAE s'accompagne d'une inflammation de la pinéale. D'abord observée chez le cobaye immunisé avec un extrait soluble total de rétine ou de pinéale de cette espèce, la pinéalite auto-immune a été ensuite produite chez lt rat, le singe et le cobaye avec l'antigène$S$, et récemment avec l'IRBP, purifiés à partir de la rétine de bœuf. On ignore actuellement si d'autres protéines communes aux $\mathrm{PR}$ et aux $\mathrm{Pi}$ possèdent cette propriété de déclencher une maladie auto-immune.

L'association de deux maladies auto-immunes provoquées par le même auto-antigène dans deux organes différents est une situation peu couranté. La pinéalite differe de l'UAE par une évolution plus précoce (peut-être liée à l'absence de barriêre hémato-encéphalique au niveau de la pinéale) et plus prolongée (inflammation chronique). De plus, la nature de l'infiltrat inflammatoire est différente : purement lymphocytaire dans la pinéale, il est riche en neutrophiles dans la rétine. L'importance de l'infiltration cellulaire de la pinéale varie selon les espèces. Chez le cobaye, elle est très sévère et peut aboutir, après plusieurs mois, à une destruction complète du parenchyme glandulaire (figure 6.

Ce modèle de pinéalite autoimmune pourrait se révéler très utile pour l'étude de la physiologie de la pinéale. Il suggère la possibilité d'une pathologie associée de la pinéale et de la rétine chez l'homme. Une inflammation de la pinéale pourrait expliquer l'existence de signes neuro- 


\section{RÉFÉRENCES}

24. Arendt J. Mammalian pineal rhythms Pineal Res Rev 1985; 3:161-213.

25. Reiter RJ. Action spectra, dose-response relationships, and temporal aspects of light's effects on the pineal gland. Ann NY Acad Sci 1985 ; 453 : 215-30.

26. Menaker M, Binkley S. Neural and endo crine control of circadian rhythms in the ver tebrates. In : Aschoff J, ed. Handbook of Behavioral Neurobiology, Vol. 4: Biological Rhythms. New York : Plenum Press, 1981 : 243-55.

27. Cassone VM, Takahashi JS, Blaha CD, Lane RF, Menaker M. Dynamics of noradrenergic circadian input to the chicken pineal gland. Brain Res. 1986 ; 384 : 334-41.

28. Voisin P, Collin JP. Regulation of chic ken pineal arylalkylamine- $\mathrm{N}$-acetyltransferase by postsynaptic $\alpha_{2}$-adrenergic receptors. Life Sci 1986 ; 39 : 2025-32.

29. Menaker M, Wisner S. Temperaturecompensated circadian clock in the pineal of Anolis. Proc Natl Acad Sci USA 1983; 80 6119-21.

30. Wurtman RJ, Waldhauser F. Melatonin in humans. $J$ Neural Transm 1986 ; 21 (suppl) $1-504$.

31. Hastings $\mathrm{MH}$, Herbert J, Martensz ND Roberts AC. Annual reproductive rhythms in mammals : mechanisms of light synchronization. Ann NY Acad Sci 1985; 453 : 183-204.

32. Pévet $P$, Buijs $R M$, Masson-Pévet $M$, Canguilhem B. Pineal, central peptidergic innervation and seasonal functions. In : Trentini GP et al., eds. Fundamentals and Clinics in Pineal Research. New York : Raven Press, 1987 (sous presse).

33. Brun J. Le dosage radioimmunologique de a mélatonine : aspects méthodologiques, intérêt et applications en pathologie psychiatrique et neurologique. Thèse Doctorat, Lyon I : Université Claude-Bernard, 1986 : 1-171.

34. Arendt J, Bojkowski C, English J, Francy C, Kemp M, Skene D. Effects and metabo lism of melatonin in humans. In : Trentini GP at al., eds. Fundamentals and Clinics in Pineal Research. New York : Raven Press, 1987 (sous presse)

35. Souêtre E, Salvati E, Darcourt G. Chronoendocrinologie de la dépression. médecine/sciences $1987 ; 3: 343-51$.

36. Kozak (de) Y, Mirshahi M, Faure JP. Rétine et auto-immunité. médecine/sciences 1987 méningés accompagnant certains types d'inflammations oculaires (uvéo-méningites, ophtalmie sympathique).

Une autre maladie commune à la rétine et à la pinéale est le rétinoblastome trilatéral, probablement dû à l'expression simultanée dans ces deux organes du gène du rétinoblastome. Cette affection reflète encore les analogies entre phototransducteurs diencéphaliques, PR et Pi.

En conclusion, les phototransducteurs de la rétine et de la pinéale, malgré leur spécialisation fonctionnelle, présentent de nombreuses convergences reconnues, tout d'abord au niveau de leur organisation, ensuite dans les mécanismes de phototransduction et de régulation de leur activité circadienne, enfin sur la nature de leurs messages. Transmise par les cellules photosensibles, l'information photopériodique - un puissant "donneur de temps" entraîne sur une période de $24 \mathrm{~h}$ les oscillateurs biologiques circadiens. Certains de ces oscillateurs gouvernent, selon divers processus, l'activité rythmique des phototransducteurs. L'expression la plus évidente de cette activité correspond à la sécrétion nocturne de la mélatonine (MEL) qui, chez les mammifères, synchronise les rythmes circadiens et circannuels. Les recherches actuelles sont orientées vers : (a) la reconnaissance d'autres facteurs externes et signaux internes susceptibles de moduler la synthèse rythmique de la MEL ; (b) l'approfondissement des acquis sur les mécanismes de régulation de cette synthèse ; (c) l'identification d'autres molécules " messages " des phototransducteurs (exemple : neuropeptides) ; (d) l'élargissement des connaissances sur le rôle de la MEL et indoles apparentés ; et (e) la détermination des cibles et mécanismes d'action de la MEL. Les données sur la MEL débouchent sur diverses perspectives médicales. Enfin, parmi les analogies établies entre photorécepteurs et pinéalocytes, certaines permettent d'élucider l'origine de pathologies communes à la rétine et à la pinéale

\section{Summary}

Retinal photoreceptors and pineal transducers of vertebrates share common or analogous features concerning (1) their functional organization, (2) the mechanisms of phototransduction, (3) the regulatory mechanisms of their circadian activity and (4) their chemical signals. Photosensitive cells transduce the photoperiodic information (a powerful Zeitgeber) into signals which entrain circadian endogenous oscillators (clocks) with a $24 \mathrm{~h}$ period. Some oscillators drive in turn the circadian activity of both the pineal transducers and the retinal photoreceptors. This rhythmic activity is reflected on the pattern of their melatonin secretion. In mammals, the nocturnal secretion of melatonin is involved in the synchronization of circadian and circannual rhythms. In humans, pineal melatonin is also secreted at night. Variations in the circadian rhythm of melatonin release in healthy humans as well as alterations of this rhythm in patients with various disorders have been studied. The role of melatonin as a synchronizer is under investigation. Analogies between diencephalic phototransducers are also found in pathology : common proteins may be auto-antigens inducing inflammatory diseases in both the retina and the pineal.

\section{Remerciements}

Les travaux de recherche ont été effectués grâce au soutien de l'Inserm, du Cnrs, de la fondation Langlois (Rennes), de la fondation pour la recherche médicale (Paris) et de l'université de Poitiers.

\section{TIRÉS A PART}

J.-P. Collin : laboratoire de biologie cellulaire et Cnrs UA 290, université de Poitiers, 40 avenue du recteur Pineau, 86022 Poitiers Cedex, France. 